\title{
Using Flow Disruptions to Examine System Safety in Robotic-Assisted Surgery: Protocol for a Stepped Wedge Crossover Design
}

Myrtede C Alfred $^{1}$, PhD; Tara N Cohen ${ }^{2}, \mathrm{PhD}$; Kate A Cohen ${ }^{2}$, MA; Falisha F Kanji ${ }^{2}$, BA; Eunice Choi ${ }^{2}$, MS; John Del Gaizo ${ }^{3}$, BSc; Lynne S Nemeth ${ }^{4}$, PhD; Alexander V Alekseyenko ${ }^{3}, \mathrm{PhD} ;$ Daniel Shouhed ${ }^{2}$, MD; Stephen J Savage ${ }^{5}$, $\mathrm{MD}$; Jennifer T Anger ${ }^{2}, \mathrm{MD}$; Ken Catchpole ${ }^{1}, \mathrm{PhD}$

\footnotetext{
${ }^{1}$ Medical University of South Carolina, Department of Anesthesia and Perioperative Medicine, Charleston, SC, United States

${ }^{2}$ Cedars-Sinai Medical Center, Department of Surgery, Los Angeles, CA, United States

${ }^{3}$ Medical University of South Carolina, Biomedical Informatics Center, Charleston, SC, United States

${ }^{4}$ Medical University of South Carolina, College of Nursing, Charleston, SC, United States

${ }^{5}$ Department of Urology, Medical University of South Carolina, Carleston, SC, United States
}

\section{Corresponding Author:}

Myrtede C Alfred, $\mathrm{PhD}$

Medical University of South Carolina

Department of Anesthesia and Perioperative Medicine

167 Ashley Avenue

Suite 300 (MSC 912)

Charleston, SC, 29425

United States

Phone: 18438761838

Email: $\underline{\text { alfredm@musc.edu }}$

\begin{abstract}
Background: The integration of high technology into health care systems is intended to provide new treatment options and improve the quality, safety, and efficiency of care. Robotic-assisted surgery is an example of high technology integration in health care, which has become ubiquitous in many surgical disciplines.

Objective: This study aims to understand and measure current robotic-assisted surgery processes in a systematic, quantitative, and replicable manner to identify latent systemic threats and opportunities for improvement based on our observations and to implement and evaluate interventions. This 5-year study will follow a human factors engineering approach to improve the safety and efficiency of robotic-assisted surgery across 4 US hospitals.
\end{abstract}

Methods: The study uses a stepped wedge crossover design with 3 interventions, introduced in different sequences at each of the hospitals over four 8-month phases. Robotic-assisted surgery procedures will be observed in the following specialties: urogynecology, gynecology, urology, bariatrics, general, and colorectal. We will use the data collected from observations, surveys, and interviews to inform interventions focused on teamwork, task design, and workplace design. We intend to evaluate attitudes toward each intervention, safety culture, subjective workload for each case, effectiveness of each intervention (including through direct observation of a sample of surgeries in each observational phase), operating room duration, length of stay, and patient safety incident reports. Analytic methods will include statistical data analysis, point process analysis, and thematic content analysis.

Results: The study was funded in September 2018 and approved by the institutional review board of each institution in May and June of 2019 (CSMC and MDRH: Pro00056245; VCMC: STUDY 270; MUSC: Pro00088741). After refining the 3 interventions in phase 1, data collection for phase 2 (baseline data) began in November 2019 and was scheduled to continue through June 2020. However, data collection was suspended in March 2020 due to the COVID-19 pandemic. We collected a total of 65 observations across the 4 sites before the pandemic. Data collection for phase 2 was resumed in October 2020 at 2 of the 4 sites.

Conclusions: This will be the largest direct observational study of surgery ever conducted with data collected on 680 robotic surgery procedures at 4 different institutions. The proposed interventions will be evaluated using individual-level (workload and attitude), process-level (perioperative duration and flow disruption), and organizational-level (safety culture and complications) 
measures. An implementation science framework is also used to investigate the causes of success or failure of each intervention at each site and understand the potential spread of the interventions.

International Registered Report Identifier (IRRID): DERR1-10.2196/25284

(JMIR Res Protoc 2021;10(2):e25284) doi: 10.2196/25284

\section{KEYWORDS}

robotic surgical procedures; patient safety; ergonomics; crossover design

\section{Introduction}

\section{Background}

The integration of technology into health care systems is intended to provide new treatment options and improve the quality, safety, and efficiency of care. Robotic-assisted surgery (RAS) is an example of high technology integration in health care, which has become ubiquitous in many surgical disciplines. RAS cases have tripled over the past decade [1] largely replacing both open and traditional laparoscopic surgeries for many common procedures [2]. Similar to many other types of technology in health care, RAS has changed tasks and workflow $[3,4]$, demanding additional skills or training and introducing new complexities ranging from skill building and learning curves to workspace and organizational issues associated with operating room (OR) layout. Although RAS is associated with less postoperative pain [5], blood loss [6], and conversion to open surgery [5], safety incidents in RAS may be higher than in traditional laparoscopy [7], which has led to concerns about the speed of adoption and implementation [8]. Similar to other advanced technologies, the spread of RAS has preceded these system-level considerations, which are difficult to predict, so risks may not be immediately apparent and often go unaddressed [9-12].

RAS implementation focuses on establishing the technical skills of the surgeon operating via the robotic console [13]. However, the physical separation of the surgeon from the OR team also introduces additional communication challenges [14,15], which can lead to errors [16] and even patient harm [17,18]. RAS has particularly acute effects on equipment congestion, the movement paths of staff, and the safe positioning of data and power cables necessary for function [19]. The learning curve required to counter this multitude of systems integration challenges may continue in RAS well beyond those required in open surgery cases $[2,19]$ and account for a steady increase in the experience recommended to achieve competency [7]. Thus, increasing task demands, combined with unique teamwork and communication challenges and existing workspace issues, may predispose to safety incidents in RAS. However, organizations are left to identify and resolve these risks without formal guidance and, in many cases, without available expertise to create formal solutions [7,20]. Human factors engineering techniques, which have been applied across many different industries to improve safety and performance [21], can be used to identify and alleviate risks in RAS. Using ethnographic approaches and systems analysis tools, human factors engineering seeks to enhance clinical performance through an understanding of the effects of teamwork, tasks, equipment, workspace, culture, and organization on human behavior and abilities.

As models of surgical processes have improved, it has become possible to reliably observe the disruptive effects of systems issues on intraoperative performance and their downstream effects on mortality and morbidity. For nearly 2 decades, direct observation of surgical work has been used to understand potential hazards in the surgical process [22,23]. Direct observation remains the best way to record variations in a process, the impact of system design on individual patterns of work, and the wider systems effects of implementing surgical technology. Unlike laboratory or simulated settings, direct observation allows us to distinguish between work as done (ie, what really happens) and work as imagined (ie, what should happen, what we think happens, or what we are told happens), illuminating the reality of how work is accomplished outside of an idealized expected or desired occurrence of events. In this paper, we discuss the design of a methodological framework and study execution applied to improve the processes of care in RAS.

\section{Study Objectives}

This 5-year study will take a human factors engineering approach to improve the safety and efficiency of RAS across 4 US hospitals. The primary objective of this study is to generate a set of integrated, evidence-based tools for improving the safety and efficiency of robotic surgery by (1) improving teamwork and communication skills, (2) improving and standardizing technical tasks such as instrument changes and robotic docking, and (3) improving the working environment. The secondary objectives are to (1) understand the effects of organizational and work context on the spread of good practice in high-technology surgery and (2) generate a computational model of the mechanisms by which small, seemingly innocuous events escalate to create serious surgical complications. This will fundamentally improve our understanding of how innovative surgical technologies can be safely deployed and integrated within clinical work systems.

\section{Methods}

\section{Study Design}

This 6-phase study includes the observation and analysis of RAS cases sampled across 4 hospitals. The study will use a pseudostepped wedge crossover design with 3 individual interventions-teamwork training (TT), task design (TD), and workspace design (WD), introduced in different sequences at each of the 4 hospital sites over 4 phases (phases 3-6) of 8 months each. We elaborate on the proposed interventions below. 


\section{TT Interventions}

TT interventions will be built based on teamwork training and nontechnical skills frameworks and will support the skills needed for teams to address RAS-specific communication challenges. The TT approach will consist of a TeamSTEPPS [24,25] driven training package (4- to 6-hour meeting for surgeons and anesthesiologists via small group teaching and successive 1-hour meetings for OR staff) complemented by on the spot coaching by human factors experts to offer reminders and encouragement.

\section{TD Interventions}

TD interventions will focus on specifying, ordering, and allocating tasks to specific roles to improve efficiency, visibility, and reliability [26]. A previously performed failure modes and effects analysis [27,28] will be used to prioritize tasks for redesign. The Systems Engineering Initiative for Patient Safety model [21] will be used to determine a human-centered systems model of each task, and task analysis will be used to define roles, sequences, and allocation. Finally, we will practice and refine these redesigns using in situ simulation trials.

\section{WD Interventions}

WD involves proposing and implementing new OR layout configurations to improve the use of space in RAS. OR layouts will be configured to ensure (1) the surgeon can see the patient and the team from the console, (2) the team can see the surgeon, (3) staff can move freely in the room, (4) robot docking can occur from multiple angles, (5) minimize cable tensions and trip hazards, and (6) optimization of OR equipment preparation and instrument storage. Key movement-oriented tasks will be used to plot ideal movement paths on existing room layouts, and new layouts will be proposed and tested to reduce unnecessary movement and disruption.

\section{Stacking Interventions}

Given the close interactions between technology, tasks, teamwork, and process [29-32], we hypothesize that multiple interventions will function synergistically. Teamwork benefits from visual cues, sightlines, and face-to-face communication $[33,34]$; TD benefits from improved teamwork to allow better coordination of complex, interdependent tasks [29,31,35]; and better efficacy of teamwork-related checklists [16], improved equipment storage, and visibility through better WD allows for improved task performance [30,36,37]. Thus, this study is designed to specifically test each of these interactions.

This design allows for sufficient implementation and sampling of the interventions, introduces individual components of an overall improvement strategy, and evaluates how each change contributes to a larger whole (Table 1).

Table 1. Study design.

\begin{tabular}{|c|c|c|c|c|c|c|c|}
\hline Project phase & Phase 1 & Phase 2 & Phase 3 & Phase 4 & Phase 5 & Phase 6 & Analysis \\
\hline Months & $1-12$ & $13-20$ & $21-28$ & $29-36$ & $37-44$ & $45-52$ & $53-60$ \\
\hline $\begin{array}{l}\text { Medical University of } \\
\text { South Carolina }\end{array}$ & $\begin{array}{l}\text { Intervention refine- } \\
\text { ment }\end{array}$ & Baseline & $\mathrm{TT}^{\mathrm{a}}$ & $\mathrm{TT}+\mathrm{TD}^{\mathrm{b}}$ & $\mathrm{TT}+\mathrm{TD}+\mathrm{WD}^{\mathrm{c}}$ & $\mathrm{TT}+\mathrm{TD}+\mathrm{WD}^{\mathrm{d}}$ & Analysis \\
\hline $\begin{array}{l}\text { Cedars-Sinai Medical } \\
\text { Center }\end{array}$ & $\begin{array}{l}\text { Intervention refine- } \\
\text { ment }\end{array}$ & Baseline & TD & $\mathrm{TD}+\mathrm{WD}$ & $\mathrm{WD}+\mathrm{TD}^{\mathrm{d}}$ & $\mathrm{TD}+\mathrm{WD}+\mathrm{TT}$ & Analysis \\
\hline Marina del Rey Hospital & $\begin{array}{l}\text { Intervention refine- } \\
\text { ment }\end{array}$ & Baseline & WD & $\mathrm{WD}^{\mathrm{d}}$ & $\mathrm{WD}+\mathrm{TT}$ & $\mathrm{WD}+\mathrm{TT}+\mathrm{TD}$ & Analysis \\
\hline $\begin{array}{l}\text { Ventura County Medical } \\
\text { Center }\end{array}$ & $\begin{array}{l}\text { Intervention refine- } \\
\text { ment }\end{array}$ & Baseline & Baseline $^{\mathrm{d}}$ & Baseline & $\mathrm{TT}+\mathrm{TD}+\mathrm{WD}$ & $\mathrm{TT}+\mathrm{TD}+\mathrm{WD}$ & Analysis \\
\hline
\end{tabular}

${ }^{\mathrm{a}} \mathrm{TT}$ : teamwork training.

${ }^{\mathrm{T}} \mathrm{TD}$ : task design.

${ }^{\mathrm{c}} \mathrm{WD}$ : workspace design.

${ }^{\mathrm{d}}$ Control phases.

\section{Power Analysis}

Using multiple regression with 10 predictor variables (4 sites, 5 data collection phases, and 3 interventions and 1 baseline period) and assuming a normal distribution, 40 observations per site per time per intervention will provide at least $80 \%$ power to find a statistically significant effect of the intervention on surgery duration. Achieving this level of statistical power remains possible with 23 observations per phase per site, making our planned sample of 40 robust, should data collection be more challenging than anticipated.

\section{Study Setting}

The study will be conducted at 4 hospitals in the United States, which include 2 tertiary centers with very different geography and demographics, a public safety net hospital, and a small private community hospital. The Medical University of South Carolina (MUSC) is an 864-bed level 1 trauma academic medical center in the southeastern United States. MUSC uses a Si da Vinci robot for general surgery procedures and $\mathrm{Xi}$ (X generation) for urology and gynecology procedures. Cedars-Sinai Medical Center (CSMC) is a large nonprofit tertiary care center with 958 beds and a level 1 trauma center designation in the western United States. CSMC currently has 7 da Vinci robots: 5 dual Xi consoles and 2 Xi single consoles. Marina del Rey Hospital (MDRH) is a 145-bed community hospital, acquired by the Cedars-Sinai Health System in 2018. The hospital has a small yet active robotic surgical program dating back to 2012. There is one Si robot that is used daily (up to 10 cases weekly). Ventura County Medical Center (VCMC) 
is a designated level 2 trauma center safety net hospital in the western United States and acquired its first da Vinci robot (Xi) in 2017, which is actively being used in general surgery, urology, and gynecology.

At MUSC, CSMC, and MDRH, we will sample from the following RAS procedures: urogynecology (sacrocolpopexy with and without hysterectomy), gynecology (hysterectomy for benign and malignant conditions), general and colorectal surgery (colon resection, abdominal wall hernia repair, hiatal hernia repair), bariatric (sleeve gastrectomy), and urology (simple and radical prostatectomy and nephrectomy). These cases are performed with enough volume to facilitate comparison through statistical analysis. At VCMC, an opportunity sampling approach, in which we collect any RAS procedure available, will be used because of the low RAS case volume.

\section{Measures}

Measures will be evaluated across 3 dimensions of RAS-individual (clinicians), process (RAS case), and system (hospital) levels - and will be collected using hospital databases, observation, surveys, and interviews (Table 2).

Table 2. Measures and administration.

Method and measures/and variables $\quad$ Phases $\quad$ Administration

\section{Extraction from hospital database}

Covariates including perioperative duration, blood Intervention+baseline loss, conversion to open returns to the $\mathrm{OR}^{\mathrm{a}}$

\section{Direct observation}

Patient details (age, BMI, ASA ${ }^{\mathrm{b}}$ )

Flow disruptions

Surgical phase duration

Oxford NOTECHS 2

Intervention adherence metric

Surveys

SURG-TLX

Safety attitudes questionnaire

Concurrent acceptability
Intervention+baseline

Intervention+baseline

Intervention+baseline

Intervention+baseline

Intervention

Intervention+baseline

Intervention+baseline

Intervention
Data collected for all patients in each intervention phase via hospital databases $(\mathrm{n}=680)$

Collected during direct observation in the OR (or retrospectively collected from patient's health record)

Direct observation of number, type, and Process rate per observation $(n \sim 27,200)$

Collected during direct observation in Process the OR

Direct observation for each phase of surgery for surgeon, OR staff, and anesthesia subteams

Direct observation once during each surgical observation (for intervention phases (3-6); $n=520)$

Completed once per observed surgery by surgeon, anesthesiologist, circulating nurse, and scrub tech (n 2270)

Administered on web via REDCap ${ }^{c}$ once System per phase for all RAS $^{\mathrm{d}}$ practitioners $(n \sim 425)$

Administration on web via REDCap [38] Individual twice per intervention phase (phases 36) for all RAS staff and clinicians (n 950)

Observations and in-person interviews conducted with a diverse sample of OR staff following the implementation of all interventions $(n=8-10$ individuals per site)

\footnotetext{
${ }^{\mathrm{a} O R}$ : operating room.

${ }^{b}$ ASA: American Society of Anesthesiologists.

${ }^{\mathrm{c}}$ REDcap: Research Electronic Data Capture.

${ }^{d}$ RAS: robotic-assisted surgery.
} 


\section{Covariates}

Contextual covariates of known influence include patient details (age, sex, BMI, American Society of Anesthesiologists [ASA] Physical Status classification), surgery details (procedure description, procedure category, date, robot model ( $\mathrm{S}, \mathrm{Si}, \mathrm{Xi}$ ), hospital, OR number, approximate room size), and personnel details (number of surgical trainees, OR staff trainees [circulating nurses and surgical technicians], and anesthesia trainees). These covariates will be collected during the observations and/or retrospectively from the patient's electronic health record. We will also record operative and in-room time, intraoperative complications, blood loss, conversion to open surgery (which requires undocking the robot and making an abdominal incision), and returns to the OR via hospital electronic records for each intervention period.

\section{Flow Disruptions}

Deviations from the natural progression of a task (ie, flow disruptions [FDs]) [39] were collected throughout all phases of each operation. Data collection includes a brief description of the event observed, time of occurrence, major category, and severity. FDs will be assigned a category and severity score during observation. With respect to classification, each FD will be assigned one of 8 possible categories: communication, coordination, equipment, training, external factors, environment, patient factors, and surgical task considerations (Table 3) based on an adapted taxonomy developed by Catchpole et al [40]. Minor categories may be developed for a more granular analysis of the data following data collection. FDs will also be assigned a severity score, ranging from 0 to 2 : (0: potential disruption to the process, 1: disruption to the process, and 2: increased patient safety risk).

Table 3. Flow disruption taxonomy.

\begin{tabular}{|c|c|c|}
\hline Category & Description & Examples \\
\hline Communication & $\begin{array}{l}\text { Any miscommunication that impacts surgery } \\
\text { progress }\end{array}$ & $\begin{array}{l}\text { Repeat information, misunderstanding, irrelevant conver- } \\
\text { sation }\end{array}$ \\
\hline Coordination & $\begin{array}{l}\text { Any lapse in teamwork to prepare for or conduct } \\
\text { surgery that affects surgery flow }\end{array}$ & $\begin{array}{l}\text { Equipment adjustment or reposition, personnel rotation, } \\
\text { personnel unavailable, lack of knowledge }\end{array}$ \\
\hline Equipment & Any equipment issue that affects surgery progress & $\begin{array}{l}\text { Robot inoperative, equipment or /instrument inoperative, } \\
\text { suture issues, insufflation problems }\end{array}$ \\
\hline Environment & Any room conditions that impact surgery progress & $\begin{array}{l}\text { Outlet positioning, untangling wires and tubing, architec- } \\
\text { tural design, lighting, noise }\end{array}$ \\
\hline External factors & $\begin{array}{l}\text { Any interruption that is not relevant to the current } \\
\text { case }\end{array}$ & $\begin{array}{l}\text { External personnel, hospital-wide alarm, personal elec- } \\
\text { tronic devices }\end{array}$ \\
\hline Patient factors & $\begin{array}{l}\text { Any patient characteristic that impedes efficient } \\
\text { surgery }\end{array}$ & $\begin{array}{l}\text { Unexpected patient reaction, patient allergy, individual } \\
\text { differences }\end{array}$ \\
\hline Surgical task considerations & $\begin{array}{l}\text { Any surgeon pauses to determine the next surgical } \\
\text { step }\end{array}$ & Surgeon decision-making, instrument changes \\
\hline Training & $\begin{array}{l}\text { Any instruction given to surgical team members } \\
\text { related to the case }\end{array}$ & $\begin{array}{l}\mathrm{OR}^{\mathrm{a}} \text { staff training, anatomy discussion, robot technical } \\
\text { instruction }\end{array}$ \\
\hline
\end{tabular}

${ }^{\mathrm{a} O R}$ : operating room.

\section{Surgical Phase Duration}

Each RAS procedure will be evaluated throughout 5 distinct surgical phases: (1) wheels in until incision, (2) incision to the surgeon on console (including the docking process), (3) surgeon on console to surgeon off console, (4) surgeon off console to patient closure, and (5) patient closure to wheels out. The duration of each phase will be recorded by the observers during data collection.

\section{Oxford NOTECHS 2}

The Oxford NOTECHS 2 [41] rating system will be used to evaluate the nontechnical skills of the OR team. The scale includes 4 dimensions-leadership and management, teamwork and cooperation, problem-solving and decision-making, and situation awareness-rated on an 8-point scale. Observers will record NOTECHS ratings for each team member during the case.

\section{Intervention Adherence Metric}

The extent to which interventions are fully used following implementation will be assessed using the intervention adherence metric [42-44], a metric developed based on the developed interventions. It will consist of a series of observational scores (Likert and check boxes) that will be deployed during each surgical observation to evaluate the use of interventions, based on observable components of each intervention. This will be deployed uniformly at baseline and all intervention phases, allowing us to understand the use of intervention during each operation.

\section{SURG-TLX}

Subjective workload ratings will be obtained using the SURG-TLX (Task Load Index) [42]. This visual-analog workload measure asks each surgical team member to select a score from 1 to 20 on 6 parameters: mental demands, physical demands, temporal demands, task complexity, situational stress, 
and distractions, which are then aggregated and rescaled to generate a workload score between 0 and 100 .

\section{Safety Attitudes Questionnaire}

Safety culture will be assessed using the Safety Attitudes Questionnaire (SAQ) [45], which has been extensively used for nearly 2 decades. The teamwork subscale has been sensitive to teamwork interventions [46], whereas the perceptions of management subscale has identified barriers to such interventions [43]. This will be administered via REDCap (Research Electronic Data Capture, a web-based Health Insurance Portability and Accountability Act-compliant survey platform) [38] in the last 2 weeks of each data collection phase to all staff involved in robotic surgery during that trial period. Subanalysis via surgical specialty and specific operations performed will allow us to track subtle changes over time.

\section{Concurrent Acceptability}

To gauge team members' responses to the interventions, we will administer the concurrent acceptability [44] measure (7 items, 5-point Likert scale) to all involved staff after the first month and at the end of the last month of each intervention phase (estimate 30-50 staff per site per phase). The measure is based on the Theoretical Framework of Acceptability model (version 2), which reflects the extent to which people deliver or receive a health systems intervention consider it to be appropriate based on anticipated or experiential cognitive and emotional responses to the intervention.

\section{Study Procedures}

\section{Observer Training}

Ensuring observers are effectively trained to perceive FDs and collect data on teamwork above the noise of otherwise normal system function is a critical requirement for this study [23]. During the prebaseline phase, observers will receive extensive training that includes initial classroom instruction (human factors and FD classification frameworks) and practice and familiarization (eg, identification of OR team members and the components of the operating room environment) in the OR with 2 human factors researchers with extensive experience with direct observation and FD measurement in surgery. Observers will be trained to understand the basic steps for each surgery type and are familiarized with the surgical subspecialties and components of the surgical robot. Trainees will also be provided with relevant reading material on FDs, NOTECHs, and RAS and given an example of a completed data collection tool.

Familiarization observations will take place across 3 stages: (1) orientation to the OR, (2) practice observations, and (3) simultaneous observation of interrater reliability (Table 4). Weekly meetings, including the observers and principal investigators, will be initiated to combat drift and allow observers to review their observations with the team.

Table 4. Observation protocol.

\begin{tabular}{|c|c|c|}
\hline Observation stage & Number of observations & Description \\
\hline Orientation & 1 & $\begin{array}{l}\text { - Trainee and trainer observe one full RAS }{ }^{\mathrm{a}} \text { procedure together. This serves to orient the } \\
\text { trainee to the OR } \\
\text { - } \quad \text { Trainer demonstrates the following behaviors: } \\
\text { - } \quad \text { Checking in with the charge nurse before entering OR } \\
\text { - } \quad \text { Checking in with circulating nurse on entry to OR } \\
\text { - } \quad \text { Where to stand in the OR and what to avoid } \\
\text { - } \quad \text { Trainer engages in postobservation discussion } \\
\text { - } \quad \text { Discussion of individuals in the room } \\
\text { - }\end{array}$ \\
\hline Practice & 3 & $\begin{array}{l}\text { - Trainee and trainer observe } 3 \text { full RAS procedures together, each using the data collec- } \\
\text { tion tool but without discussing their observations with one another } \\
\text { - } \quad \text { Debrief after surgery } \\
\text { rently, trainee checks observations they caught and discussion of those that they did } \\
\text { not }\end{array}$ \\
\hline $\begin{array}{l}\text { Interrater reliabili- } \\
\text { ty }\end{array}$ & 5 & $\begin{array}{l}\text { - } \quad \text { Trainee and trainer observe } 5 \text { observations for interrater reliability } \\
\text { If } \text { IRR }^{\mathrm{d}}(\mathrm{kappa}>0.7) \text { observers were considered trained and they could observe indepen- } \\
\text { dently }\end{array}$ \\
\hline
\end{tabular}

\footnotetext{
${ }^{\mathrm{a}} \mathrm{RAS}$ : robotic-assisted surgery.

${ }^{\mathrm{b}} \mathrm{OR}$ : operating room.

${ }^{\mathrm{c}} \mathrm{FD}$ : flow disruption.

${ }^{d}$ IRR: interrater reliability.
} 


\section{In-Services}

Before conducting observations, 15-minute in-services will be conducted with the staff on each unit at each study site to explain the research, introduce them to the research team, and allow them to ask questions and express their concerns. In-services will be led by a human factors expert and surgeon team member(s). Furthermore, an information sheet will be provided to staff to educate them about the purpose of the study and provide contact information for members of the study team whether they have any questions.

\section{Data Collection}

\section{Observations}

A total of 4 trained human factors researchers will observe 680 RAS cases over the course of the study period. For MUSC, CSMC, and MDRH, observers will capture 40 cases during each of the 5 data collection phases (phases 2-6, each 8 months in duration). For VCMC, 40 cases will be captured in each of 2 phases: baseline (which spans across phases 2-4) and intervention (phases 5 and 6; Table 5).

Observers will collect FDs, NOTECH ratings, and all relevant case-related covariates, including patient details (age, sex, BMI, ASA classification), surgery details (procedure description, date, hospital, OR number, room size), personnel details (number of surgical trainees by type, OR staff trainees, and anesthesia trainees by type), and robot details ( $\mathrm{S}, \mathrm{Si}$, or $\mathrm{Xi}$ model). During the intervention phases, the intervention adherence metric will also be collected during each surgical observation to evaluate the use of interventions.

Field notes will also be collected monthly by the observers at each of the 4 sites. Field notes generally consist of 2 parts: descriptive and reflective information. Descriptive information attempts to accurately document factual data (eg, date and time) and the settings, actions, behaviors, and conversations observed. Reflective information documents your thoughts, ideas, questions, and concerns as you are conducting the observation. These notes will provide additional context for the implementation of the intervention using the Consolidated Framework for Implementation Research (CFIR) [47].

Data will be collected in the OR using Microsoft Surface Pro 6 tablets. Urban Armor Gear Hand Strap \& Shoulder Strap Military Drop Tested Cases are also used to provide ergonomic support and handling of tablets for observers standing or seated on stools for long period. XCOREsion $15-45$ by J-Go Tech Microsoft Surface Portable Chargers were given to each observer to provide external battery life when collecting data over 2 or more consecutive cases with no opportunity to charge their tablets between cases.

Table 5. Data collection schedule.

\begin{tabular}{|c|c|c|c|c|c|c|}
\hline Project phase & Phase 2 & Phase 3 & Phase 4 & Phase 5 & Phase 6 & Total \\
\hline $\begin{array}{l}\text { Medical Universi- } \\
\text { ty of South Caroli- } \\
\text { na }\end{array}$ & 40 (5 per month) & 40 (5 per month) & 40 (5 per month) & 40 (5 per month) & 40 (5 per month) & 200 \\
\hline $\begin{array}{l}\text { Cedars-Sinai } \\
\text { Medical Center }\end{array}$ & 40 (5 per month) & 40 ( 5 per month $)$ & 40 (5 per month) & 40 (5 per month) & 40 (5 per month) & 200 \\
\hline $\begin{array}{l}\text { Marina del Rey } \\
\text { Hospital }\end{array}$ & 40 (5 per month) & 40 (5 per month) & 40 (5 per month) & 40 (5 per month) & 40 (5 per month) & 200 \\
\hline $\begin{array}{l}\text { Ventura County } \\
\text { Medical Center }\end{array}$ & 13 (1-2 per month) & 14 (1-2 per month) & 13 (1-2 per month) & 20 (2-3 per month) & 20 (2-3 per month) & 80 \\
\hline Total & 133 & 134 & 133 & 140 & 140 & 680 \\
\hline
\end{tabular}

\section{Surveys}

The SURG-TLX will be collected in person during direct observation and will be administered on a Microsoft Excel form located on the observer's Microsoft Surface Pro tablets. The SAQ and concurrent acceptability will each be collected via a REDCap survey emailed to surgeons and OR staff.

\section{Postimplementation Evaluation}

We will evaluate interventions as multiple case studies using in-depth interviews and observations to gain an understanding of how these process changes are adapted in each setting and what facilitates success and barriers to these changes. A diverse sample of OR managers, nurses, surgeons, assistants, and technical support personnel $(n=8-10$ individuals per site) will be interviewed using semistructured interview guides to elicit narratives of individual experiences surrounding RAS implementation, teamwork, surgical safety, and facilitators and /barriers to successful RAS workflow. Interviews will be guided by the CFIR [47] to examine how the interventions were implemented in each setting, considering intervention characteristics, inner and outer context, and characteristics of individuals and process involved. Our qualitative analysis will examine convergence and divergence of narratives and will present those in a case study approach. An Olympus voice recorder will be used, and audio files will be professionally transcribed. Interview transcripts and field notes will be uploaded to NVivo (QSR International, Victoria, Australia), a qualitative and mixed methods analysis software, for the analysis.

\section{Data Analysis}

\section{Statistical Analysis}

We will use multivariable regression models to explore the relationship between the covariates (ie, site, specialty, BMI, and teamwork) and process measures (ie, FDs and durations), examining how these relationships are modified by 
interventions. The following are the specific questions we seek to answer: (1) What interventions are used (intervention adherence metric)? (2) Did OR staff like the interventions (concurrent acceptability)? (3) Did the interventions change attitudes (SAQ)? (4) Did the interventions change individual workload (TLX) and/or improve teamwork (NOTECHS)? (5) Did the interventions result in a better process (FD)? and (6) Did the interventions reduce surgical durations and/or blood loss and/or OR returns? Statistical analysis will be conducted using the $\mathrm{R}$ programming language (R CORE TEAM, version 3.5.2) and assessed at the significance level of $\alpha .05$.

\section{Point Process Analysis}

Direct observation of surgical processes may be useful in modeling adverse event causation by looking at the concatenation of smaller, seemingly innocuous errors to larger, more clinically serious situations [48-50]. The primary purpose of the proposed analyses is to develop a quantitative framework that allows for the evaluation of the snowball hypothesis. The rationale behind this hypothesis is that accidents and injuries arise from the sequence of multiple, frequently occurring individual errors. Adverse outcomes can be seen both as the unlucky coincidence of multiple randomly occurring errors and/or as a causative chain of events where one error leads to the next, creating an error cascade (or snowball). A range of exploratory Markov chain, Poisson process, and changepoint modeling techniques will be applied with the R programming language to analyze data across more than 1000 procedures and identify error causation mechanisms as random coincidences or as a deterministic error cascade. This mode of analysis aims to be a profound advance, not solely in understanding and addressing surgical complications and adverse events but in the entire way in which accidents are viewed.

\section{Intervention Analysis}

An inductive and deductive thematic content analysis approach will be used to analyze the qualitative data [51]. In total, 2 research team members will individually code all interviews and field notes, first in a deductive pass, using a codebook to tag segments of text specific to the CFIR constructs and systems model concepts. Next, an inductive pass through the data will identify new concepts to develop themes that have not been previously identified. Codes will identify causes, explanations, relationships, patterns, and themes related to the implementation of new RAS workflows. After iterative analyses, the 2 coders will immerse and crystallize [52] the final set of themes, confirm these findings with the research team, and develop a case study of comprehensive user experiences that promote successful implementation of RAS.

\section{Results}

\section{Funding and Ethics}

The study was funded in September 2018 and approved by the institutional review board of each institution in May and June of 2019 (CSMC and MDRH: Pro00056245; VCMC: STUDY 270; MUSC: Pro00088741).

\section{Data Collection}

After refining the 3 interventions in phase 1, data collection for phase 2 (baseline data) began in November 2019 and was scheduled to continue through June 2020. However, data collection was suspended in March 2020 due to the COVID-19 pandemic. We collected a total of 65 observations across the 4 sites before the pandemic. Data collection for phase 2 was resumed in October 2020 at 2 of our 4 sites.

\section{Discussion}

\section{Overview}

The overall goal of our research involves conducting multiple system-level interventions in RAS to validate a methodological approach to understanding and addressing latent systemic threats from new surgical technologies and measure both the effects of improvements that result as well as the utility of the interventions. Multiple interventions will be developed, tested, and planned to substantially expand our understanding of surgical safety in high-technology health care settings. This project will be the most comprehensive study to apply a human factors framework to study safety and efficiency, as it relates to technology integration in surgery. Although focused on RAS, the proposed observational, implementation, and evaluative methods of this study can be successfully applied to other health care settings integrating advanced technological systems. The study aims to address challenges and concerns using a mixed methods approach, including interviews, observations, work systems approaches, longitudinal ethnographic sampling techniques, and statistical modeling. This design is intended to capture the etiology of failure modes resulting from the mismatch between technology and existing culture. The combination of approaches will allow us to address how small, otherwise innocuous incidents can snowball into accidents and injuries in health care settings $[48,49,53,54]$. We also apply an implementation science framework to understand barriers to implementation, particularly of distal influences [55] or where staff may not always be supportive [7]. Understanding how and why observed effects differ among settings will allow for improved spread and sustainability. Implementing and sustaining improvements requires an ongoing involvement of stakeholders across organizational levels and boundaries [56].

Our sample includes a high volume of RAS cases performed using the da Vinci robot and conveniently sampled; this will limit the range of surgical procedures observed and will likely result in an unbalanced sample across the 4 sites. Scheduling is complex, and case cancelations and delays are an inherent deficiency in collecting observational data. The presence of the observer impacts the nature of data collected, whether as a result of implicit bias or obstructed views, and will thus affect how the data are analyzed. Although the methods described earlier are imperfect, future research teams may explore better ways to conduct these types of studies, such as through the use of video monitoring and other innovative approaches.

\section{Limitations}

Although direct observation provides a unique opportunity to gain a true understanding of the current state of the system [16], 
there are challenges in conducting observational research in health care settings. These challenges include the time and effort required to train observers and organize observations [17], the costs of employing researchers to conduct observations, and the potential for the Hawthorne effect [18]. In addition, good interrater reliability among observers needs to be established and maintained throughout the course of the study, and observers need to be supported, as they may view traumatic events and feel unwelcomed in the OR [23]. Previous research has used video capture and remote video monitoring to identify teamwork, communication, and other challenges in the OR and RAS [57]. However, these methods introduce logistic and ethical challenges, including institutional review board concerns related to the identifiability of participants and data capture of adverse events. Poor fidelity and recording quality, limited viewing angles, and obstructions may also limit the usefulness of the recorded data. Moreover, the use of video recording still requires that observers conduct several videos to record observations, possibly extending the time required and expenses associated with data collection. Video capture and observations together, as was used by Randell et al [57], may represent the most comprehensive approach.

\section{Conclusions}

This project will demonstrate the value of understanding technologies in the wild; the nature of partnerships between human factors experts, clinicians, administrators, and OR staff; the integration and understanding of surgical technologies; and the implications for future technological development and clinical practice. Ultimately, this study will fundamentally improve our understanding of how innovative surgical technologies can be safely deployed and integrated into complex clinical work systems. We welcome the development of similar methodologies for the evaluation and integration of various kinds of technology in health care.

\section{Acknowledgments}

This project was funded under grant number HS026491-01 from the Agency for Healthcare Research and Quality, US Department of Health and Human Services.

\section{Authors' Contributions}

TC, LN, AA, JA, and KC conceptualized and designed the study and interventions. SS, DS, and JA facilitated OR access and data collection. MA, JG, KC, FK, and EC collected data under the supervision of TC, KC, and AA. MA, TC, and KC wrote the first draft, and the manuscript was edited by KC, LN, JA, DS, and SS. All remaining authors reviewed, provided feedback, and approved the final manuscript.

\section{Conflicts of Interest}

None declared.

\section{References}

1. Navaratnam A, Abdul-Muhsin H, Humphreys M. Updates in Urologic Robot Assisted Surgery. F1000Res 2018;7 [FREE Full text] [doi: 10.12688/f1000research.15480.1] [Medline: 30613380]

2. Catchpole K, Perkins C, Bresee C, Solnik MJ, Sherman B, Fritch J, et al. Safety, efficiency and learning curves in robotic surgery: a human factors analysis. Surg Endosc 2016 Sep 16;30(9):3749-3761. [doi: 10.1007/s00464-015-4671-2] [Medline: 26675938]

3. Catchpole KR. Task, team and technology integration in the paediatric cardiac operating room. Progress in Pediatric Cardiology 2011 Dec;32(2):85-88. [doi: 10.1016/j.ppedcard.2011.10.005]

4. Pennathur PR, Thompson D, Abernathy JH, Martinez EA, Pronovost PJ, Kim GR, et al. Technologies in the wild (TiW): human factors implications for patient safety in the cardiovascular operating room. Ergonomics 2013 Feb;56(2):205-219. [doi: 10.1080/00140139.2012.757655] [Medline: 23384283]

5. Anger JT, Mueller ER, Tarnay C, Smith B, Stroupe K, Rosenman A, et al. Robotic Compared With Laparoscopic Sacrocolpopexy. Obstetrics \& Gynecology 2014;123(1):5-12. [doi: 10.1097/aog.0000000000000006]

6. Anderson JE, Chang DC, Parsons JK, Talamini MA. The first national examination of outcomes and trends in robotic surgery in the United States. J Am Coll Surg 2012 Jul;215(1):107-114. [doi: 10.1016/j.jamcollsurg.2012.02.005] [Medline: $\underline{22560318]}$

7. Mirheydar HS, Parsons JK. Diffusion of robotics into clinical practice in the United States: process, patient safety, learning curves, and the public health. World J Urol 2013 Jun 29;31(3):455-461. [doi: 10.1007/s00345-012-1015-x] [Medline: $\underline{23274528]}$

8. Kirkner RM. Rush to robotic surgery outpaces medical evidence, critics say. Manag Care 2014 May;23(5):26-9, 33 [FREE Full text] [Medline: 25016847]

9. Bainbridge L. Ironies of automation. Analysis, design and evaluation of man-machine systems: Elsevier 1983:129-135. [doi: 10.1016/b978-0-08-029348-6.50026-9]

10. Cook RI, Woods DD. Adapting to new technology in the operating room. Hum Factors 1996 Dec;38(4):593-613. [doi: 10.1518/001872096778827224] [Medline: $\underline{\text { 8976623] }}$ 
11. Lee JD, See KA. Trust in Automation: Designing for Appropriate Reliance. Human Factors 2004;46(1):50-80. [doi: 10.1518/hfes.46.1.50.30392]

12. Parasuraman R, Riley V. Humans and Automation: Use, Misuse, Disuse, Abuse. Human Factors 2016 Nov 23;39(2):230-253. [doi: 10.1518/001872097778543886]

13. Kim J, Anger JT. Is robotic sacrocolpopexy a marketing gimmick or a technological advancement? Current Opinion in Urology 2010;20(4):280-284. [doi: 10.1097/mou.0b013e32833aaa2b]

14. Catchpole KR, Hallett E, Curtis S, Mirchi T, Souders CP, Anger JT. Diagnosing barriers to safety and efficiency in robotic surgery. Ergonomics 2018 Jan 08;61(1):26-39 [FREE Full text] [doi: 10.1080/00140139.2017.1298845] [Medline: 28271956]

15. Randell R, Honey S, Alvarado N, Pearman A, Greenhalgh J, Long A, et al. Embedding robotic surgery into routine practice and impacts on communication and decision making: a review of the experience of surgical teams. Cogn Tech Work 2016 Apr 1;18(2):423-437. [doi: 10.1007/s10111-016-0368-0]

16. Lingard L, Regehr G, Orser B, Reznick R, Baker GR, Doran D, et al. Evaluation of a preoperative checklist and team briefing among surgeons, nurses, and anesthesiologists to reduce failures in communication. Arch Surg 2008 Jan;143(1):12-17. [doi: 10.1001/archsurg.2007.21] [Medline: 18209148]

17. Greenberg CC, Regenbogen SE, Studdert DM, Lipsitz SR, Rogers SO, Zinner MJ, et al. Patterns of communication breakdowns resulting in injury to surgical patients. J Am Coll Surg 2007 Apr;204(4):533-540. [doi:

10.1016/j.jamcollsurg.2007.01.010] [Medline: 17382211]

18. Rogers SO, Gawande AA, Kwaan M, Puopolo AL, Yoon C, Brennan TA, et al. Analysis of surgical errors in closed malpractice claims at 4 liability insurers. Surgery 2006 Jul;140(1):25-33. [doi: 10.1016/j.surg.2006.01.008] [Medline: 16857439]

19. Zorn K, Wille M, Thong A, Katz M, Shikanov S, Razmaria A, et al. Continued improvement of perioperative, pathological and continence outcomes during 700 robot-assisted radical prostatectomies. Canadian Journal of Urology 2009;16(4).

20. Waterson P, Catchpole K. Human factors in healthcare: welcome progress, but still scratching the surface. BMJ Qual Saf 2016 Jul 18;25(7):480-484. [doi: 10.1136/bmjqs-2015-005074] [Medline: 26685148]

21. Carayon P, Schoofs Hundt A, Karsh B, Gurses AP, Alvarado CJ, Smith M, et al. Work system design for patient safety: the SEIPS model. Qual Saf Health Care 2006 Dec;15 Suppl 1:i50-i58 [FREE Full text] [doi: 10.1136/qshc.2005.015842] [Medline: 17142610$]$

22. Carthey J, de Leval MR, Reason JT. The human factor in cardiac surgery: errors and near misses in a high technology medical domain. The Annals of Thoracic Surgery 2001 Jul;72(1):300-305. [doi: 10.1016/s0003-4975(00)02592-3]

23. Catchpole K, Neyens DM, Abernathy J, Allison D, Joseph A, Reeves ST. Framework for direct observation of performance and safety in healthcare. BMJ Qual Saf 2017 Dec;26(12):1015-1021 [FREE Full text] [doi: 10.1136/bmjqs-2016-006407] [Medline: 28971880]

24. Clancy CM. TeamSTEPPS: optimizing teamwork in the perioperative setting. AORN J 2007 Jul;86(1):18-22. [doi: 10.1016/j.aorn.2007.06.008] [Medline: 17621444]

25. Sheppard F, Williams M, Klein VR. TeamSTEPPS and patient safety in healthcare. J Healthc Risk Manag 2013 Jan 18;32(3):5-10. [doi: 10.1002/jhrm.21099] [Medline: 23335296]

26. Berber E, Engle KL, Garland A, String A, Foroutani A, Pearl JM, et al. A critical analysis of intraoperative time utilization in laparoscopic cholecystectomy. Surg Endosc 2001 Feb 3;15(2):161-165. [doi: 10.1007/s004640000329] [Medline: $\underline{11285960]}$

27. Lago P, Bizzarri G, Scalzotto F, Parpaiola A, Amigoni A, Putoto G, et al. Use of FMEA analysis to reduce risk of errors in prescribing and administering drugs in paediatric wards: a quality improvement report. BMJ Open 2012 Dec 18;2(6):e001249 [FREE Full text] [doi: 10.1136/bmjopen-2012-001249] [Medline: 23253870]

28. Schriefer J, Leonard MS. Patient safety and quality improvement: an overview of QI. Pediatr Rev 2012 Aug 01;33(8):353-9; 359. [doi: 10.1542/pir.33-8-353] [Medline: 22855927]

29. Morgan L, New S, Robertson E, Collins G, Rivero-Arias O, Catchpole K, et al. Effectiveness of facilitated introduction of a standard operating procedure into routine processes in the operating theatre: a controlled interrupted time series. BMJ Qual Saf 2015 Feb 03;24(2):120-127. [doi: 10.1136/bmjqs-2014-003158] [Medline: 25368320]

30. McCulloch P, Morgan L, New S, Catchpole K, Roberston E, Hadi M, et al. Combining Systems and Teamwork Approaches to Enhance the Effectiveness of Safety Improvement Interventions in Surgery. Annals of Surgery 2017;265(1):90-96. [doi: 10.1097/sla.0000000000001589]

31. Catchpole K. Task, team and technology integration in the paediatric cardiac operating room. Progress in Pediatric Cardiology. 2011. URL: https://doi.org/10.1016/j.ppedcard.2011.10.005 [accessed 2021-01-11]

32. Holden RJ, Carayon P, Gurses AP, Hoonakker P, Hundt AS, Ozok AA, et al. SEIPS 2.0: a human factors framework for studying and improving the work of healthcare professionals and patients. Ergonomics 2013 Nov;56(11):1669-1686 [FREE Full text] [doi: 10.1080/00140139.2013.838643] [Medline: 24088063]

33. Randell R, Alvarado N, Honey S, Greenhalgh J, Gardner P, Gill A, et al. Impact of Robotic Surgery on Decision Making: Perspectives of Surgical Teams. AMIA Annu Symp Proc 2015;2015:1057-1066 [FREE Full text] [Medline: 26958244]

34. Hilligoss B, Cohen MD. The unappreciated challenges of between-unit handoffs: negotiating and coordinating across boundaries. Ann Emerg Med 2013 Feb;61(2):155-160. [doi: 10.1016/j.annemergmed.2012.04.009] [Medline: 22560466] 
35. Catchpole KR, de Leval MR, McEwan A, Pigott N, Elliott MJ, McQuillan A, et al. Patient handover from surgery to intensive care: using Formula 1 pit-stop and aviation models to improve safety and quality. Paediatr Anaesth 2007 May;17(5):470-478. [doi: 10.1111/j.1460-9592.2006.02239.x] [Medline: 17474955]

36. McCulloch PS, Kreckler S, New S, Sheena Y, Handa A, Catchpole K. Effect of a "Lean" intervention to improve safety processes and outcomes on a surgical emergency unit. BMJ 2010 Nov 02;341:c5469. [doi: 10.1136/bmj.c5469] [Medline: 21045024]

37. Farrokhi FR, Gunther M, Williams B, Blackmore CC. Application of Lean Methodology for Improved Quality and Efficiency in Operating Room Instrument Availability. J Healthc Qual 2015;37(5):277-286. [doi: 10.1111/jhq.12053] [Medline: 24112283]

38. Harris PA, Taylor R, Thielke R, Payne J, Gonzalez N, Conde JG. Research electronic data capture (REDCap)--a metadata-driven methodology and workflow process for providing translational research informatics support. J Biomed Inform 2009 Apr;42(2):377-381 [FREE Full text] [doi: 10.1016/j.jbi.2008.08.010] [Medline: 18929686]

39. Wiegmann DA, ElBardissi AW, Dearani JA, Daly RC, Sundt TM. Disruptions in surgical flow and their relationship to surgical errors: an exploratory investigation. Surgery 2007 Nov;142(5):658-665. [doi: 10.1016/j.surg.2007.07.034] [Medline: 17981185]

40. Catchpole K, Perkins C, Bresee C, Solnik MJ, Sherman B, Fritch J, et al. Safety, efficiency and learning curves in robotic surgery: a human factors analysis. Surg Endosc 2016 Sep 16;30(9):3749-3761. [doi: 10.1007/s00464-015-4671-2] [Medline: $\underline{26675938]}$

41. Mishra A, Catchpole K, McCulloch P. The Oxford NOTECHS System: reliability and validity of a tool for measuring teamwork behaviour in the operating theatre. Qual Saf Health Care 2009 Apr 01;18(2):104-108. [doi: 10.1136/qshc. 2007.024760] [Medline: 19342523 ]

42. Wilson MR, Poolton JM, Malhotra N, Ngo K, Bright E, Masters RSW. Development and validation of a surgical workload measure: the surgery task load index (SURG-TLX). World J Surg 2011 Sep 20;35(9):1961-1969 [FREE Full text] [doi: 10.1007/s00268-011-1141-4] [Medline: 21597890]

43. Catchpole KR, Dale TJ, Hirst DG, Smith JP, Giddings TA. A Multicenter Trial of Aviation-Style Training for Surgical Teams. Journal of Patient Safety 2010;6(3):180-186. [doi: 10.1097/pts.0b013e3181f100ea]

44. Sekhon M, Cartwright M, Francis JJ. Acceptability of healthcare interventions: an overview of reviews and development of a theoretical framework. BMC Health Serv Res 2017 Jan 26;17(1):88 [FREE Full text] [doi: 10.1186/s12913-017-2031-8] [Medline: 28126032]

45. Sexton JB, Thomas EJ, Helmreich RL. Error, stress, and teamwork in medicine and aviation: cross sectional surveys. BMJ 2000 Mar 18;320(7237):745-749 [FREE Full text] [doi: 10.1136/bmj.320.7237.745] [Medline: 10720356]

46. McCulloch P, Mishra A, Handa A, Dale T, Hirst G, Catchpole K. The effects of aviation-style non-technical skills training on technical performance and outcome in the operating theatre. Qual Saf Health Care 2009 Apr 01;18(2):109-115. [doi: 10.1136/qshc.2008.032045] [Medline: 19342524]

47. Damschroder LJ, Aron DC, Keith RE, Kirsh SR, Alexander JA, Lowery JC. Fostering implementation of health services research findings into practice: a consolidated framework for advancing implementation science. Implement Sci 2009 Aug 07;4(1):1-15 [FREE Full text] [doi: 10.1186/1748-5908-4-50] [Medline: 19664226]

48. de Leval MR, Carthey J, Wright DJ, Farewell VT, Reason JT. Human factors and cardiac surgery: A multicenter study. The Journal of Thoracic and Cardiovascular Surgery 2000 Apr;119(4):661-672. [doi: 10.1016/s0022-5223(00)70006-7]

49. Woolf SH, Kuzel AJ, Dovey SM, Phillips RL. A string of mistakes: the importance of cascade analysis in describing, counting, and preventing medical errors. Ann Fam Med 2004 Jul 01;2(4):317-326 [FREE Full text] [doi: 10.1370/afm.126] [Medline: 15335130$]$

50. Catchpole KR, Giddings AEB, de Leval MR, Peek GJ, Godden PJ, Utley M, et al. Identification of systems failures in successful paediatric cardiac surgery. Ergonomics 2006 Feb 20;49(5-6):567-588. [doi: 10.1080/00140130600568865] [Medline: 16717010$]$

51. Elo S, Kyngäs H. The qualitative content analysis process. J Adv Nurs 2008 Apr;62(1):107-115. [doi: 10.1111/j.1365-2648.2007.04569.x] [Medline: 18352969]

52. Crabtree BF, Miller WL. Doing qualitative research. London: Sage; 1999.

53. Woods DE. How Unexpected Events Produce An Escalation Of Cognitive And Coordinative Demands. In: Stress, Workload and Fatigue. Hillsdale, NJ: Lawrence Erlbaum; 2004:327-333.

54. Hofer TP, Hayward RA. Are bad outcomes from questionable clinical decisions preventable medical errors? A case of cascade iatrogenesis. Ann Intern Med 2002 Sep 03;137(5 Part 1):327-333 [FREE Full text] [doi:

10.7326/0003-4819-137-5 part 1-200209030-00008] [Medline: 12204016]

55. Dixon-Woods M, Pronovost PJ. Patient safety and the problem of many hands. BMJ Qual Saf 2016 Jul 24;25(7):485-488 [FREE Full text] [doi: 10.1136/bmjqs-2016-005232] [Medline: 26912578]

56. Blandford A, Berndt E, Catchpole K, Furniss D, Mayer A, Mentis H, et al. Strategies for conducting situated studies of technology use in hospitals. Cogn Tech Work 2014 Dec 16;17(4):489-502. [doi: 10.1007/s10111-014-0318-7] 
57. Randell R, Honey S, Hindmarsh J, Alvarado N, Greenhalgh J, Pearman A, et al. A realist process evaluation of robot-assisted surgery: integration into routine practice and impacts on communication, collaboration and decision-making. Health Serv Deliv Res 2017 Jun;5(20):1-140. [doi: 10.3310/hsdr05200] [Medline: 28813131]

\author{
Abbreviations \\ ASA: American Society of Anesthesiologists \\ CFIR: Consolidated Framework for Implementation Research \\ CSMC: Cedars-Sinai Medical Center \\ FD: flow disruptions \\ MDRH: Marina del Rey Hospital \\ MUSC: Medical University of South Carolina \\ OR: operating room \\ RAS: robotic-assisted surgery \\ SAQ: Safety Attitudes Questionnaire \\ TD: task design \\ TLX: Task Load Index \\ TT: teamwork training \\ VCMC: Ventura County Medical Center \\ WD: workspace design
}

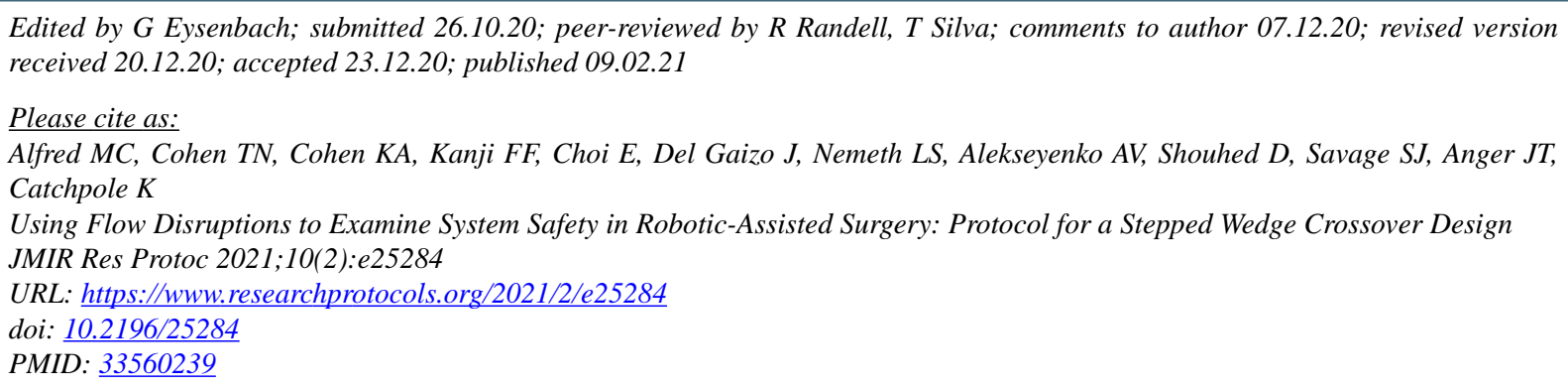

CMyrtede C Alfred, Tara N Cohen, Kate A Cohen, Falisha F Kanji, Eunice Choi, John Del Gaizo, Lynne S Nemeth, Alexander V Alekseyenko, Daniel Shouhed, Stephen J Savage, Jennifer T Anger, Ken Catchpole. Originally published in JMIR Research Protocols (http://www.researchprotocols.org), 09.02.2021. This is an open-access article distributed under the terms of the Creative Commons Attribution License (https://creativecommons.org/licenses/by/4.0/), which permits unrestricted use, distribution, and reproduction in any medium, provided the original work, first published in JMIR Research Protocols, is properly cited. The complete bibliographic information, a link to the original publication on http://www.researchprotocols.org, as well as this copyright and license information must be included. 